\title{
CODES FROM UNIT GROUPS OF DIVISION ALGEBRAS OVER NUMBER FIELDS
}

\author{
by \\ Christian Maire \& Aurel Page
}

\begin{abstract}
Lenstra and Guruswami described number field analogues of the algebraic geometry codes of Goppa. Recently, the first author and Oggier generalised these constructions to other arithmetic groups: unit groups in number fields and orders in division algebras; they suggested to use unit groups in quaternion algebras but could not completely analyse the resulting codes. We prove that the noncommutative unit group construction yields asymptotically good families of codes for the sum-rank metric from division algebras of any degree, and we estimate the size of the alphabet in terms of the degree.
\end{abstract}

\section{Introduction}

Number field codes, introduced by Lenstra [9] and independently rediscovered by Guruswami [5], are number field analogues of the geometric codes of Goppa 4 built from curves over finite fields. In these original constructions, the codes are constructed from the ring of integers of a number field.

In [11, the first author and Oggier extended these ideas: they explained how to construct codes from any arithmetic group, and they analysed the parameters of the resulting codes in the case of the unit group of the ring of integers of a number field, and in the case of an order in a division algebra. In every case, it was possible to obtain asymptotically good families of codes using towers of number fields with bounded root discriminant. The multiplicative group of an order in a quaternion algebra was also considered with a partial analysis and the question of constructing asymptotically good codes from these groups was left open, short of having adapted techniques, as explained in Remark 12 of [11].

We completely analyse the noncommutative unit group codes in any degree, by using a metric that is adapted to those groups. We also measure the minimal distance of the code in terms of the sum-rank distance introduced by Martínez-Peñas 13, which also yields Hamming distance codes by using the columns of the matrices as the alphabet.

2000 Mathematics Subject Classification. - 94B40, 11R52, 11T71, 94B75.

Key words and phrases. - Number Field Codes, Division Algebras over Number Fields, Asymptotically Good Codes.

The authors would like to thank the Warwick Mathematics Department and the Department of Mathematics at Cornell University for providing a stimulating research atmosphere. We also thank Xavier Caruso for suggesting the use of the sum-rank distance, and an anonymous referee for their valuable feedback. We also thank the EPSRC for financial support via the EPSRC Programme Grant EP/K034383/1 LMF: L-functions and modular forms. CM was also partially supported by the Region Bourgogne Franche-Comté, the ANR project FLAIR (ANR-17-CE40-0012) and the EIPHI Graduate School (ANR-17-EURE-0002). 
We analyse how the size of the alphabet varies with the degree of the algebra, and we prove the following theorem.

Theorem. - For all $d \geq 2$, there exists a family of asymptotically good number field codes for the sum-rank distance, each obtained from the group of units of reduced norm 1 in a maximal order in a division algebra of degree $d$, over a fixed alphabet $\mathrm{M}_{d}\left(\mathbb{F}_{p}\right)$, where $\log p=c \log d+O(\log \log d)$ and $c>0$ is a constant.

We did not try to get the sharpest possible bounds, but the asymptotic of $\log p$ in terms of $d$ is probably correct apart from the value of the constant $c$. Our analysis uses tools from the theory of arithmetic groups: the metric we choose is closely related to the canonical metric on the associated symmetric space, and we use Macdonald's [10] and Prasad's [17] volume formulas. We also rely on a classical integration formula in $K A K$ coordinates for the Haar measure of a semisimple Lie group (see for instance [8, Proposition 5.28]), but the analysis of the dependence on the degree required computing normalisation factors that we could not find in the literature. Our method should generalise to arithmetic lattices in other semisimple groups.

One nice feature of the noncommutative case is that the formula for the covolume of the arithmetic group is nicer than the one for the regulator of a number field, since the relevant values of the zeta function are evaluations at integers greater than 1 . For fixed $d$, it is even possible to give closed formulas for the parameters of the code using our techniques, and we carry out these computations in the case $d=2$. This is why we derive exact formulas whenever possible, and then deduce an asymptotic analysis of the interesting quantities.

For the sake of comparison, we revisit the additive case and carry out the analysis of the dependence on the degree. We obtain the following result: although the additive and multiplicative groups have very different geometry, the asymptotic behaviour of the code is the same.

Theorem. - For all $d \geq 2$, there exists a family of asymptotically good number field codes for the sum-rank distance, each obtained from the additive group of a maximal order in a division algebra of degree $d$, over a fixed alphabet $\mathrm{M}_{d}\left(\mathbb{F}_{p}\right)$, where $\log p=$ $\frac{1}{2} \log d+O(\log \log d)$.

In the context of coding theory, our results are mostly of theoretical value, for two reasons. First, the code construction is not effective in its current form (see Lemma 1, the element $c \in G$ in the definition of the code is not explicit); it seems like an interesting problem to derive an effective version of our construction and an efficient decoding algorithm. Second, the notion of asymptotically good codes is rather coarse; it would be valuable to prove more precise estimates, maybe in special cases in the spirit of our Section 5.4, since fixing the degree allows one to get explicit formulas for many relevant parameters. We hope that our results shed some extra light on the family of Goppa-style constructions, and that they provide a starting ground for the study of related codes, for instance over function fields, by changing the groups, by optimising the shape of the ball (B in the notations of Section 2), or by considering polyalphabetic versions of our construction.

The article is organised as follows. We first recall, in Section 2, the general construction of arithmetic group codes following Maire-Oggier [11], and we review the basic properties 
of central simple algebras over number fields in Section 3. In Section 4, we carry out the crucial volume computations that we need to estimate the parameters of the codes. We analyse the multiplicative construction in Section 5, where we prove our main theorem and give a detailed study of the quaternion case. Finally, we revisit the additive construction in Section 6 .

\section{The construction}

We recall the general construction as in [11]. Given

(i) a locally compact group $G$ and a compact subset $\mathrm{B} \subset G$,

(ii) a lattice $\Gamma \subset G$, i.e. a discrete subgroup with a fundamental domain of finite Haar measure,

(iii) a map $\Theta: \Gamma \rightarrow \mathcal{A}^{N}$, where $\mathcal{A}$ is an alphabet (i.e. a finite set) and $N \geq 1$ is an integer,

we consider a code $\mathcal{C}=\Theta(\Gamma \cap c \mathrm{~B})$, where $c \in G$ is such that $|\Gamma \cap c \mathrm{~B}|$ is maximal. There may be more than one such $c$; we simply pick an arbitrary $c$. Codewords of $\mathcal{C}$ are elements of $\mathcal{A}^{N}$. The main tool to estimate the rate of such codes is an idea of Lenstra, which we express in the following lemma.

Lemma 1. - Let $\mu$ be a Haar measure on $G$. If $\left.\Theta\right|_{\Gamma \cap c \mathrm{~B}}$ is injective, then

$$
|\mathcal{C}| \geq \frac{\mu(\mathrm{B})}{\mu(G / \Gamma)}
$$

Proof. - This is [11, Lemma 1].

One natural way to construct such a code from an arithmetic group is as follows. Given a number field $F$, a linear algebraic group $\mathbb{G}$ defined over $F$ and an arithmetic group $\Gamma \subset$ $\mathbb{G}\left(\mathbb{Z}_{F}\right)$, we can consider $G=\mathbb{G}\left(F \otimes_{\mathbb{Q}} \mathbb{R}\right)$ in which $\Gamma$, under a mild extra condition on $\mathbb{G}$, is a lattice via the natural embedding $\Gamma \subset \mathbb{G}(F) \subset G$, by a theorem of Borel and HarishChandra [1]. Then define

$$
\Theta: \Gamma \rightarrow \prod_{\mathfrak{p} \in S} \mathbb{G}\left(\mathbb{Z}_{F} / \mathfrak{p}\right) \rightarrow \mathcal{A}^{N},
$$

where $S$ is a finite set of prime ideals of $F$ and $\mathcal{A}$ is related to the groups $\mathbb{G}\left(\mathbb{Z}_{F} / \mathfrak{p}\right)$. For instance, if for all $\mathfrak{p} \in S$ we have an embedding $\mathbb{G}\left(\mathbb{Z}_{F} / \mathfrak{p}\right) \hookrightarrow \mathrm{GL}_{d}\left(\mathbb{F}_{q_{0}}\right)$, then we can take $\mathcal{A}=\mathrm{M}_{d}\left(\mathbb{F}_{q_{0}}\right)$, or $\mathcal{A}=\mathbb{F}_{q_{0}}^{d}$ in which case the map $\Theta$ picks the columns of the corresponding matrices.

We describe the interesting parameters of such codes. The length of a code $\mathcal{C} \subset \mathcal{A}^{N}$ is $N$, and we let $q=|\mathcal{A}|$. The Hamming distance $d_{H}(x, y)$ between two elements $x, y \in \mathcal{A}^{N}$ is the number of components in which they differ. The rate of $\mathcal{C}$ is $\frac{\log _{q}|\mathcal{C}|}{N}$ with $\log _{q}(t)=$ $\log t / \log q$, and the minimum Hamming distance $d_{H}(\mathcal{C})$ of $\mathcal{C}$ is the minimum Hamming distance among all pairs of distinct codewords.

Families of codes $\left(\mathcal{C}_{i}\right)_{i}$ with length $N_{i} \rightarrow \infty$ that satisfy

$$
\liminf _{i} \frac{\log _{q}\left|\mathcal{C}_{i}\right|}{N_{i}}>0, \text { and } \liminf _{i} \frac{d_{H}\left(\mathcal{C}_{i}\right)}{N_{i}}>0,
$$

are called asymptotically good codes (see for example [19] for a good explanation of the notion in the context of algebraic geometry codes). 
In the case of matrix alphabets, Martínez-Peñas 13 introduced a finer distance: the sum-rank distance. Assume $\mathcal{A}=\mathrm{M}_{d}(\mathbb{F})$ where $\mathbb{F}$ is a finite field; if $\mathcal{C} \subset \mathcal{A}^{N_{0}}$ is a code, then there is an attached code $\mathcal{C}_{\text {col }} \subset \mathcal{A}^{\prime N_{0} d}$ where $\mathcal{A}^{\prime}=\mathbb{F}^{d}$, obtained by picking the columns of the matrices. The length of the code $\mathcal{C}$ is defined to be $N=N_{0} \cdot d$ and we let $q=|\mathbb{F}|^{d}$, so that they match the corresponding notions for $\mathcal{C}_{c o l}$. The sum-rank distance $d_{R}(x, y)$ between two elements $x, y \in \mathcal{A}^{N_{0}}$ is

$$
d_{R}(x, y)=\sum_{i=1}^{N_{0}} \operatorname{rk}\left(x_{i}-y_{i}\right)
$$

Note that we have $d_{R}(x, y) \leq d_{H}(x, y)$ since the rank of a matrix is bounded by the number of nonzero columns. The rate of $\mathcal{C}$ is $\frac{\log _{q}|\mathcal{C}|}{N}$, and the minimum sum-rank distance $d_{R}(\mathcal{C})$ of $\mathcal{C}$ is the minimum sum-rank distance among all pairs of distinct codewords. Families of codes $\left(\mathcal{C}_{i}\right)_{i}$ with length $N_{i} \rightarrow \infty$ that satisfy

$$
\liminf _{i} \frac{\log _{q}\left|\mathcal{C}_{i}\right|}{N_{i}}>0, \text { and } \liminf _{i} \frac{d_{R}\left(\mathcal{C}_{i}\right)}{N_{i}}>0,
$$

are called asymptotically good codes for the sum-rank distance. If $\mathcal{C}_{i}$ is asymptotically good for the sum-rank distance, then $\left(\mathcal{C}_{i}\right)_{c o l}$ is asymptotically good (for the Hamming distance).

In the arithmetic case considered above, the code length $N$ is equal to $d \cdot|S|$, and $q=q_{0}^{d}$. The reason why we should expect arithmetic group codes to have large minimum distance is the following. If two elements $\gamma_{1}, \gamma_{2} \in \Gamma$ correspond to codewords that are close for $d_{R}$ or $d_{H}$, then they will be congruent modulo many prime ideals $\mathfrak{p}$, and therefore $\gamma_{1}^{-1} \gamma_{2}$ will be large in the real embeddings, which will contradict the fact that they are bounded by the compact set B.

We are going to consider two instances of this construction: one where $\mathbb{G}$ is the group of units of reduced norm one of a division algebra, and one where $\mathbb{G}$ is the additive group of a division algebra. The precise specification of the code is given in Section 5 for the multiplicative case and Section 6 for the additive case.

\section{Central Simple Algebras: what we need}

Our main references are [16 and [18, however the literature is abundant. All our rings will be associative and unital, and all our algebras will be finite-dimensional. We write $A^{\times}$ for the units of a ring $A$. If $R$ is a commutative ring and $d \geq 1$ an integer, let $\mathrm{M}_{d}(R)$ be the $d \times d$ matrix algebra with coefficients in $R$.

3.1. Generalities. - Let $A$ be an algebra over a field $F$. The centre of $A$ is $\mathrm{Z}(A)=$ $\{a \in A \mid x a=a x, \forall x \in A\}$. We say that $A$ is central if $\mathrm{Z}(A)=F$, that $A$ is a division algebra if $A^{\times}=A \backslash\{0\}$, and that $A$ is simple if the only two-sided ideals of $A$ are $\{0\}$ and $A$. Every division algebra is simple. The dimension of a central simple algebra over $F$ is a square $d^{2}$, and $d$ is called the degree of $A$ over $F$. A central simple algebra of degree 2 is called a quaternion algebra.

Let $A$ be a central simple algebra of degree $d$ over a field $F$. There exists a finite extension $L / F$ and an isomorphism $A \otimes_{F} L \cong \mathrm{M}_{d}(L)$ of algebras over $L$; let $\varphi$ be such an isomorphism. For all $x \in A$, the determinant $\operatorname{det} \varphi(x)$ (resp. the trace $\operatorname{tr} \varphi(x)$ ) is in $F$ and is independent of $L$ and $\varphi$; it is called the reduced norm $\operatorname{nrd}(x)$ (resp. the 
reduced trace $\operatorname{trd}(x))$ of $x$. The map $\operatorname{trd}: A \rightarrow F$ is $F$-linear, the map nrd: $A \rightarrow F$ is multiplicative, and $A^{\times}=\{x \in A \mid \operatorname{nrd}(x) \neq 0\}$.

Example 2. - Let $\mathbb{H}$ be the algebra of Hamiltonian quaternions:

$$
\mathbb{H}=\mathbb{R}+\mathbb{R} i+\mathbb{R} j+\mathbb{R} k \text { with } i^{2}=j^{2}=k^{2}=i j k=-1 .
$$

Then $\mathbb{H}$ is a central division algebra over $\mathbb{R}$. For all $x, y, z, t \in \mathbb{R}$ we have $\operatorname{nrd}(x+y i+$ $z j+t k)=x^{2}+y^{2}+z^{2}+t^{2}$ and $\operatorname{trd}(x+y i+z j+t k)=2 x$.

3.2. Over number fields. - Let $F$ be a number field of degree $n$ over $\mathbb{Q}$. Denote by $\mathbb{Z}_{F}$ the ring of integers of $F$, and by $\mathbb{P}_{\infty}$ the set of infinite places of $F$. If $I \subset \mathbb{Z}_{F}$ is a nonzero ideal of $\mathbb{Z}_{F}$, let $\mathrm{N}(I)=\left|\mathbb{Z}_{F} / I\right|$ be its norm. Let $A$ be a central simple algebra of degree $d$ over $F$.

3.2.1. - Let $x \in A$. Then the absolute norm $\mathrm{N}(x) \in \mathbb{Q}$ of $x$ is the absolute value of the determinant of the matrix of left multiplication by $x$ on $A$, seen as a matrix in $\mathrm{M}_{d^{2} n}(\mathbb{Q})$. We have $\mathrm{N}(x)=\left|N_{F / \mathbb{Q}}(\operatorname{nrd}(x))^{d}\right|$, where $N_{F / \mathbb{Q}}$ denotes the norm in $F / \mathbb{Q}$. When $A$ is a division algebra, $\mathrm{N}(x)=0$ if and only if $x=0$.

3.2.2. - An order in $A$ is a subring $\mathcal{O} \subset A$ that is finitely generated as a $\mathbb{Z}$-module and such that $\mathcal{O} F=A$. If $\mathcal{O}$ is an order and $x \in \mathcal{O}$ is such that $\mathrm{N}(x) \neq 0$, then $\mathrm{N}(x)=$ $|\mathcal{O} / x \mathcal{O}|$.

Let $\mathcal{O}$ be a maximal order (i.e. not properly contained in a larger order), and let $\mathfrak{p}$ be a prime ideal of $\mathbb{Z}_{F}$; let $q=\mathrm{N}(\mathfrak{p})$. We say that

- $\mathfrak{p}$ is unramified in $A$ if $\mathcal{O} / \mathfrak{p} \mathcal{O} \cong \mathrm{M}_{d}\left(\mathbb{F}_{q}\right)$;

- $\mathfrak{p}$ is ramified in $A$ otherwise.

The number of primes that ramify in $A$ is finite.

Let $\mathfrak{p}$ be a prime ideal of $\mathbb{Z}_{F}$, and denote by $F_{\mathfrak{p}}$ the completion of $F$ at $\mathfrak{p}$. Then there exists an isomorphism

$$
A \otimes_{F} F_{\mathfrak{p}} \cong \mathrm{M}_{d_{\mathfrak{p}}}\left(D_{\mathfrak{p}}\right),
$$

where $D_{\mathfrak{p}}$ is a central division algebra over $F_{\mathfrak{p}}$; let us write $\operatorname{dim}_{F_{\mathfrak{p}}} D_{\mathfrak{p}}=e_{\mathfrak{p}}^{2}$. We then have $e_{\mathfrak{p}} d_{\mathfrak{p}}=d$, the prime $\mathfrak{p}$ is ramified if and only if $e_{\mathfrak{p}}>1$, and more generally we have $\mathcal{O} / \mathfrak{P} \cong \mathrm{M}_{d_{\mathfrak{p}}}\left(\mathbb{F}_{q^{e \mathfrak{p}}}\right)$ where $\mathfrak{P}$ is the unique maximal two-sided ideal of $\mathcal{O}$ containing $\mathfrak{p}$. Let $\sigma \in \mathbb{P}_{\infty}$ be an infinite place of $F$. If $\sigma$ is complex, then there is an isomorphism

$$
A \otimes_{F} F_{\sigma} \cong \mathrm{M}_{d}(\mathbb{C}),
$$

and in this case, we say that $\sigma$ is unramified. If $\sigma$ is real, then there is an isomorphism

- $A \otimes_{F} F_{\sigma} \cong \mathrm{M}_{d}(\mathbb{R})$, in which case we say that $\sigma$ is unramified in $A$, or

- $A \otimes_{F} F_{\sigma} \cong \mathrm{M}_{d / 2}(\mathbb{H})$, in which case we say that $\sigma$ is ramified in $A$.

In all cases, we fix an isomorphism extending $\sigma$ as above, and still call it $\sigma: A \otimes_{F} F_{\sigma} \cong$ $\mathrm{M}_{d_{\sigma}}\left(D_{\sigma}\right)$, where $D_{\sigma}$ is a central division algebra of degree $e_{\sigma} \in\{1,2\}$ over $F_{\sigma}$, and we write $n_{\sigma}=\left[F_{\sigma}: \mathbb{R}\right]$.

3.2.3. - We denote by $\Delta_{F}$ the absolute value of the discriminant of $F$ and by $\operatorname{rd}_{F}$ its root discriminant $\Delta_{F}^{1 / n}$. Recall that if $K / F$ is an unramified extension of number fields then $\operatorname{rd}_{K}=\operatorname{rd}_{F}$. The reduced discriminant $\delta_{A}$ of $A$ is defined as

$$
\delta_{A}=\prod_{\mathfrak{p}} \mathfrak{p}^{d\left(1-1 / e_{\mathfrak{p}}\right)}=\prod_{\mathfrak{p}} \mathfrak{p}^{d-d_{\mathfrak{p}}}
$$


where the product is over all prime ideals of $\mathbb{Z}_{F}$. The absolute discriminant $\Delta_{A}$ of $A$ is defined as

$$
\Delta_{A}=\Delta_{F}^{d^{2}} \mathrm{~N}\left(\delta_{A}\right)^{d}
$$

\section{Volumes}

4.1. Cartan decomposition. - Let $D$ be a division algebra over $\mathbb{R}$, so that $D$ is isomorphic to one of $\mathbb{R}, \mathbb{C}$ or $\mathbb{H}$. We write $F=\mathrm{Z}(D)$ the centre of $D$, e the degree of $D$ over $F$, and $n=[F: \mathbb{R}]$. Hence: $e=n=1$ if $D=\mathbb{R} ; e=1, n=2$ if $D=\mathbb{C}$; and $e=2$, $n=1$ if $D=\mathbb{H}$. We will later apply the results of this section to the completion $\mathrm{M}_{d}(D)$ of a central simple algebra over a number field at a real or complex place $\sigma$, and the notations for those degrees will become $e_{\sigma}, n_{\sigma}$ and $d_{\sigma}$.

There exists a unique $\mathbb{R}$-linear and anti-multiplicative involution $x \mapsto \bar{x}$ on $D$ such that for all $x \in D^{\times}$we have $x \bar{x} \in \mathbb{R}_{>0} \subset D$ : it is called the canonical involution of $D$. Explicitly, the canonical involution is the identity on $\mathbb{R}$, the complex conjugation on $\mathbb{C}$, and the quaternionic conjugation on $\mathbb{H}$. In this section, we fix $d \geq 1$.

Consider the semisimple Lie group

$$
G=\mathrm{SL}_{d}(D)=\left\{g \in \mathrm{M}_{d}(D) \mid \operatorname{nrd}(g)=1\right\},
$$

the following maximal compact subgroup of $G$

$$
K=\mathrm{SU}_{d}(D)=\left\{g \in G \mid g^{t} \bar{g}=\mathrm{Id}\right\},
$$

and define

$$
A=\left\{\left(\begin{array}{ccc}
\exp \left(a_{1}\right) & & 0 \\
& \ddots & \\
0 & & \exp \left(a_{d}\right)
\end{array}\right): a_{1}, \ldots, a_{d} \in \mathbb{R} \mid \sum_{i=1}^{d} a_{i}=0\right\} \subset G .
$$

Example 3. - If $D=\mathbb{H}$ and $d=2$, then

$$
\mathrm{SL}_{d}(D)=\mathrm{SL}_{2}(\mathbb{H})=\left\{g \in \mathrm{M}_{2}(\mathbb{H}) \mid \operatorname{nrd}(g)=1\right\},
$$

where for $a, b, c, d \in \mathbb{H}$ we have

$$
\operatorname{nrd}\left(\begin{array}{ll}
a & b \\
c & d
\end{array}\right)= \begin{cases}\operatorname{nrd}(a d) & \text { if } c=0 \\
\operatorname{nrd}\left(a c^{-1} d c-b c\right) & \text { if } c \neq 0\end{cases}
$$

Note that the set $\left\{\left(\begin{array}{ll}a & b \\ c & d\end{array}\right) \in \mathrm{M}_{2}(\mathbb{H}) \mid a d-b c=1\right\}$ is not stable under multiplication.

In this case we have

$$
K=\mathrm{SU}_{d}(D)=\mathrm{SU}_{2}(\mathbb{H})=\left\{\left(\begin{array}{ll}
a & b \\
c & d
\end{array}\right) \in \mathrm{M}_{2}(\mathbb{H}) \mid a \bar{a}+b \bar{b}=c \bar{c}+d \bar{d}=1, a \bar{c}+b \bar{d}=0\right\} .
$$

Let $\mathrm{Z}_{K}(A)=\{a \in A \mid a k=k a \forall k \in K\}$ be the centraliser of $A$ in $K$. Explicitly, we have

- if $D=\mathbb{R}$, then $\mathrm{Z}_{K}(A) \cong\{ \pm 1\}^{d-1}$ is the group of diagonal matrices with coefficients \pm 1 on the diagonal and determinant 1 ;

- if $D=\mathbb{C}$, then $\mathrm{Z}_{K}(A) \cong \mathrm{U}(1)^{d-1}$ is the group of diagonal matrices with coefficients in $\mathbb{C}$ of absolute value 1 on the diagonal and determinant 1 ; 
- if $D=\mathbb{H}$, then $\mathrm{Z}_{K}(A) \cong \mathrm{SL}_{1}(\mathbb{H})^{d} \cong \mathrm{SU}_{2}(\mathbb{C})^{d}$ is the group of diagonal matrices with coefficients in $\mathbb{H}$ of reduced norm 1 on the diagonal.

Let us recall the Cartan decomposition of $G$ that will be useful for the computation of the different volumes (see for example [8, Chapter V, §4]).

Theorem 4 (Cartan decomposition). - Let $G=\mathrm{SL}_{d}(D), K$ and $A$ be as above. Then we have $G=K A K$. In the decomposition $g=k_{1} a k_{2}$ of an element $g \in G$ with $k_{i} \in K$ and $a=\operatorname{diag}\left(\exp \left(a_{1}\right), \ldots, \exp \left(a_{d}\right)\right) \in A$, the $a_{i}$ are unique up to permutation. Moreover, let $S \subset G$ be the subset of elements $g \in G$ such that the $a_{i}$ are distinct. Then for all $g$ in $S$, the pair $\left(k_{1}, k_{2}\right)$ is uniquely determined up to changing $\left(k_{1}, k_{2}\right)$ into $\left(k_{1} z^{-1}, z k_{2}\right)$ with $z \in \mathrm{Z}_{K}(A)$.

Remark 5. - When $D=\mathbb{R}$ or $\mathbb{C}$, this is also known as the singular value decomposition. Note that $G \backslash S$ has zero measure, so that we can and will restrict to $S$ when computing integrals.

Define the function $\rho: G \rightarrow \mathbb{R}_{\geq 0}$ such that for all $g \in G$ with Cartan decomposition $g=$ $k_{1} a k_{2}$, where $a=\operatorname{diag}\left(\exp \left(a_{1}\right), \ldots, \exp \left(a_{d}\right)\right)$, we have

$$
\rho(g)=\max _{i}\left|a_{i}\right| \text {. }
$$

This is well-defined since the $a_{i}$ are unique up to permutation. Note that we have $\rho\left(g^{-1}\right)=$ $\rho(g)$ for all $g \in G$.

Let us give a series of corollaries of Theorem 4 that will be useful to estimate the minimal Hamming distance of the multiplicative codes.

Let $\|\cdot\|_{2}: D^{d} \rightarrow \mathbb{R}$ be the norm on $D^{d}$ defined by $\|x\|_{2}=\left(\sum_{i=1}^{d} x_{i} \bar{x}_{i}\right)^{1 / 2}$ for all $x \in D^{d}$, and let $\|\mid\| \cdot \|: \mathrm{M}_{d}(D) \rightarrow \mathbb{R}_{\geq 0}$ be the corresponding operator norm, that is:

$$
\|g\|=\sup _{x \neq 0} \frac{\|g \cdot x\|_{2}}{\|x\|_{2}} \text { for all } g \in \mathrm{M}_{d}(D) .
$$

Corollary 6. - For all $g \in G$, we have

$$
\rho(g)=\log \max \left(\|g\|\|,\| g^{-1}\|\|\right) .
$$

Proof. - As $\|\cdot\|_{2}$ is bi-invariant by $K$, in Cartan decomposition (Theorem 4) we have $\|g\|=\max _{i} \exp \left(a_{i}\right)$. Applying this to $g$ and $g^{-1}$ gives the result.

Corollary 7. - For all $g, h \in G$, we have $\rho(g h) \leq \rho(g)+\rho(h)$.

Proof. - This follows from Corollary 6 and the submultiplicativity of operator norms.

Corollary 8. - For all $g \in G$, we have

$$
|\operatorname{nrd}(g-1)| \leq 2^{d} \exp (d \rho(g)) .
$$

Proof. - Let $x=g-1$. We claim that we have $|\operatorname{nrd}(x)| \leq\|\mid x\|^{d}$. To see this, let $V=D^{d}$ viewed as an $\mathbb{R}$-vector space of dimension $d e^{2} n$. The absolute value of the determinant of $x$ viewed as an endomorphism of $V$ is $|\operatorname{nrd}(x)|^{n e^{2}}$. So multiplication by $x$ scales volumes by $|\operatorname{nrd}(x)|^{n e^{2}}$; applying this to a ball for the norm $\|\cdot\|_{2}$, noting that the volume of the 
ball of radius $R$ is proportional to $R^{d e^{2} n}$ and using the definition of the operator norm proves the claim.

On the other hand we have

$$
\|\| x\|\leq\| g \|+1 \leq 2 \max (1,\|g\| \|) \leq 2 \exp (\rho(g)),
$$

where the last inequality follows from Corollary 6 .

4.2. Haar measure on $\mathrm{SL}_{d}(D)$. - If $M, N \in \mathrm{M}_{d}(D)$, we write $[M, N]$ for the Lie bracket $M N-N M$, and if $g \in \mathrm{GL}_{d}(D)$, we write $\operatorname{Ad}(g) M=g M g^{-1}$. The Lie algebra of $G=\mathrm{SL}_{d}(D)$ is $\mathfrak{g}=\mathfrak{s l}_{d}(D)=\left\{X \in \mathrm{M}_{d}(D) \mid \operatorname{trd}(X)=0\right\}$. We equip the $\mathbb{R}$-vector space $\mathfrak{s l}_{d}(D)$ with the positive definite inner product $(X, Y) \mapsto \operatorname{tr}_{\mathrm{Z}(D) / \mathbb{R}} \operatorname{trd}\left({ }^{t} \bar{X} Y\right)$, with corresponding norm $\|X\|^{2}=n \operatorname{trd}\left({ }^{t} \bar{X} X\right)$. This gives $G=\mathrm{SL}_{d}(D)$ the structure of a Riemannian manifold with a metric on $G$ that is invariant under left translations by arbitrary elements of $G$ and under right translation by elements of $K$. In particular, this defines a volume form $d \mu$ on $G$, on $K$ and on $\mathrm{Z}_{K}(A)$.

Let us start with the computation of the volume of $\mathrm{Z}_{K}(A)$ with respect to $d \mu$.

Lemma 9. - Let $G=\mathrm{SL}_{d}(D)$ and $K, A$ be as above. Then $\mu\left(\mathrm{Z}_{K}(A)\right)$ equals

- $2^{d-1}$ when $D=\mathbb{R}$,

- $(2 \sqrt{2} \pi)^{d-1} \sqrt{d}$ when $D=\mathbb{C}$,

- $\left(4 \sqrt{2} \pi^{2}\right)^{d}$ when $D=\mathbb{H}$.

\section{Proof. -}

- If $D=\mathbb{R}$, the group $\mathrm{Z}_{K}(A) \cong\{ \pm 1\}^{d-1}$ is finite and the corresponding measure is the counting measure.

- If $D=\mathbb{C}$, the group $\mathrm{Z}_{K}(A)$ is a product of circles $U(1)^{d-1}$, which we parametrise as the image of $[0,2 \pi]^{d-1}$ under the map

$$
\left(\theta_{1}, \ldots, \theta_{d-1}\right) \mapsto \operatorname{diag}\left(\exp \left(i \theta_{1}\right), \ldots, \exp \left(i \theta_{d-1}\right), \exp \left(-i \sum_{k=1}^{d-1} \theta_{k}\right)\right) .
$$

The Gram matrix of the corresponding tangent vectors is the $(d-1) \times(d-1)$ matrix

$$
\left(\begin{array}{cccc}
4 & 2 & \ldots & 2 \\
2 & 4 & \ddots & \vdots \\
\vdots & \ddots & \ddots & 2 \\
2 & \ldots & 2 & 4
\end{array}\right)
$$

which has determinant $2^{d-1} d$. So the corresponding volume is $(2 \pi)^{d-1} \sqrt{2^{d-1} d}$.

- If $D=\mathbb{H}$, the group $\mathrm{Z}_{K}(A)$, as a Riemannian manifold, is a direct product of $d$ copies of $\mathrm{SL}_{1}(\mathbb{H})=\left\{x+y i+z j+t k \mid x^{2}+y^{2}+z^{2}+t^{2}=1\right\}$, which is a 3-dimensional sphere of radius $\sqrt{2}$ with our normalisation. It is well-known that the volume of a 3 -dimensional sphere of radius $R$ is $2 \pi^{2} R^{3}$, giving the formula.

More generally, we can compute volumes using the following formula: 
Proposition 10. - Let $G=\mathrm{SL}_{d}(D)$ and $K, A$ be as above, and let $f \in L^{1}(G)$. Then the integral $\int_{G} f d \mu$ equals

$$
\frac{(n e)^{\frac{d-1}{2}} \sqrt{d}}{\mu\left(\mathrm{Z}_{K}(A)\right)} \int_{K \times K} \int_{a_{i}} \prod_{1 \leq i<j \leq d} \sinh \left(a_{i}-a_{j}\right)^{n e^{2}} f\left(k_{1} a k_{2}\right) d a_{i} d k_{1} d k_{2},
$$

where:

- $a=\operatorname{diag}\left(\exp \left(a_{1}\right), \ldots, \exp \left(a_{d}\right)\right)$, and

- the inner integral is over the set

$$
\left\{\left(a_{1}, \ldots, a_{d-1}\right) \in \mathbb{R}^{d-1} \mid a_{1}>a_{2}>\cdots>a_{d-1}>-\sum_{i=1}^{d-1} a_{i}\right\}
$$

and $a_{d}=-\sum_{i=1}^{d-1} a_{i}$.

Proof. - We will obtain the formula by pulling back the metric along the map

$$
\Psi: K \times A \times K \rightarrow G
$$

and computing the pullback by using the decomposition of $\mathfrak{s l}_{d}(D)$ according to restricted roots of $A$, as in [7], keeping track of all constants. We write $\mathfrak{a}$ (resp. $\mathfrak{k}$ ) for the Lie algebra of $A$ (resp. of $K)$.

First we compute the differential of $\Psi$. Let $x=\left(k_{1}, a, k_{2}\right) \in K \times A \times K$, where $a=$ $\operatorname{diag}\left(\exp \left(a_{i}\right)\right)$. Using the canonical isomorphism between the tangent space of a Lie group at an arbitrary point and its Lie algebra, we obtain that the differential of $\Psi$ at $\left(k_{1}, a, k_{2}\right)$ is the map $d \Psi_{x}: \mathfrak{k} \times \mathfrak{a} \times \mathfrak{k} \rightarrow \mathfrak{g}$ that sends

$$
\left(X_{1}, Y, X_{2}\right) \mapsto \operatorname{Ad}\left(k_{2}^{-1}\right) \operatorname{Ad}\left(a^{-1}\right) X_{1}+Y+X_{2} .
$$

To compute the pull-back of the volume form to $K \times A \times K$, it is enough to compute $\Delta_{x}=\operatorname{det}\left(d \Psi_{x}{ }^{t}\left(d \Psi_{x}\right)\right)^{1 / 2}$. We compute this determinant by blocks. The term in $\mathfrak{a}$ does not contribute. We compute the other terms by decomposing $\mathrm{M}_{d}(D)$ in $2 \times 2$ blocks corresponding to rows and columns $i$ and $j$. Consider the matrices

$$
F_{+}=\left(\begin{array}{ll}
0 & 1 \\
1 & 0
\end{array}\right) \text { and } F_{-}=\left(\begin{array}{cc}
0 & 1 \\
-1 & 0
\end{array}\right) \in \mathrm{M}_{2}(D) .
$$

We have $\operatorname{Ad}(a) F_{+}=\cosh \left(a_{i}-a_{j}\right) F_{+}+\sinh \left(a_{i}-a_{j}\right) F_{-}$and $\operatorname{Ad}(a) F_{-}=\cosh \left(a_{i}-a_{j}\right) F_{-}+$ $\sinh \left(a_{i}-a_{j}\right) F_{+}$. In addition, let $D^{0}=\{z \in D \mid \bar{z}+z=0\}$. Then $\mathfrak{k}$ is the sum over all the blocks of $F_{-} \mathbb{R} \oplus F_{+} D^{0}$, and of $Z_{\mathfrak{k}}(\mathfrak{a})$, and those are orthogonal to $F_{+} \mathbb{R}$ and $F_{-} D^{0}$. Using this, we see that the matrix of $d \Psi_{x}$ is a direct sum of blocks of the form

$$
\left(\begin{array}{ll}
\sinh \left(a_{i}-a_{j}\right) & 0 \\
\cosh \left(a_{i}-a_{j}\right) & 1
\end{array}\right)
$$

each appearing with multiplicity $\operatorname{dim}_{\mathbb{R}} D=n e^{2}$, and of the block (1 1 ) appearing with multiplicity $\left(e^{2}-1\right) d$. We therefore have

$$
\Delta_{x}=2^{\frac{\left(e^{2}-1\right) d}{2}} \prod_{1 \leq i<j \leq d} \sinh \left(a_{i}-a_{j}\right)^{n e^{2}} .
$$


The map $\Psi$ is not injective, and by Theorem 4 , outside of a set of zero measure, the fibre above $k_{1} a k_{2}$ is $\left\{\left(k_{1} z, a, z^{-1} k_{2}\right): z \in Z_{K}(A)\right\}$. With the same computation we obtain that the volume of this fibre is $2^{\frac{\left(e^{2}-1\right) d}{2}} \mu\left(Z_{K}(A)\right)$. This gives

$$
\int_{G} f d \mu=\frac{1}{\mu\left(\mathrm{Z}_{K}(A)\right)} \int_{K \times K} \int_{a \in A^{+}} \prod_{1 \leq i<j \leq d} \sinh \left(a_{i}-a_{j}\right)^{n e^{2}} f\left(k_{1} a k_{2}\right) d a d k_{1} d k_{2},
$$

where $A^{+} \subset A$ is the subset of diagonal matrices with decreasing entries. We parametrise

$$
A^{+}=\left\{\operatorname{diag}\left(\exp \left(a_{i}\right)\right):\left(a_{1}, \ldots, a_{d-1}\right) \in \mathbb{R}^{d-1} \mid a_{1}>a_{2}>\cdots>a_{d} \text { and } a_{d}=-\sum_{i=1}^{d-1} a_{i}\right\},
$$

and the factor corresponding to this second change of variables is the square root of the determinant of the Gram matrix of the tangent vectors $\operatorname{diag}(0, \ldots, 1,0, \ldots, 0,-1) \in \mathfrak{g}$. This Gram matrix is the $(d-1) \times(d-1)$ matrix

$$
n e\left(\begin{array}{cccc}
2 & 1 & \ldots & 1 \\
1 & 2 & \ddots & \vdots \\
\vdots & \ddots & \ddots & 1 \\
1 & \ldots & 1 & 2
\end{array}\right)
$$

and it has determinant $(n e)^{d-1} d$.

Proposition 11. - Let $K$ be as above. Then we have

$$
\mu(K)=\kappa \prod_{k=1}^{r} \frac{\pi^{m_{k}+1}}{m_{k} !},
$$

where

(i) if $D=\mathbb{R}$ :

- if $d$ is even, then $r=d / 2, \kappa=2^{d^{2} / 2-d / 4}$ and

$$
m_{k}=2 k-1 \text { for } k \leq r-1 \text { and } m_{r}=r-1 \text {; }
$$

- if $d$ is odd, then $r=(d-1) / 2, \kappa=2^{d^{2} / 2+d / 4-3 / 4}$ and

$$
m_{k}=2 k-1 \text { for all } k \leq r
$$

(ii) if $D=\mathbb{C}$, then $r=d-1, \kappa=2^{d^{2}+d / 2-3 / 2} \sqrt{d}$ and

$$
m_{k}=k \text { for all } k \leq r ;
$$

(iii) if $D=\mathbb{H}$, then $r=d, \kappa=2^{2 d^{2}+d / 2}$ and

$$
m_{k}=2 k-1 \text { for all } k \leq r .
$$

Proof. - We apply Macdonald's formula [10]. Recall (see [3, Part II, §19]) that the roots of $\mathfrak{k}$ with respect to $\mathfrak{t}$ are the nonzero morphisms $\alpha \in \operatorname{Hom}_{\mathbb{R}}(\mathfrak{t}, \mathbb{C})$ such that there exists a nonzero $X_{\alpha} \in \mathfrak{k} \otimes \mathbb{C}$ such that $\left[t, X_{\alpha}\right]=\alpha(t) X_{\alpha}$ for all $t \in \mathfrak{t}$; for each root $\alpha$, the attached coroot $\alpha^{\vee} \in \mathfrak{t} \otimes \mathbb{C}$ is the unique element $\alpha^{\vee} \in \mathbb{C} \cdot\left[X_{\alpha}, X_{-\alpha}\right]$ such that $\left[\alpha^{\vee}, X_{\alpha}\right]=2 X_{\alpha}$ and $\left[\alpha^{\vee}, X_{-\alpha}\right]=-2 X_{-\alpha}$. In each case, we give the Lie algebra $\mathfrak{k}$ of $K$, and in Macdonald's notations, $\mathfrak{t} \subset \mathfrak{k}, \mathfrak{t}_{\mathbb{Z}} \subset \mathfrak{t}$, and the list of roots $\alpha$, their corresponding root vectors $X_{\alpha}$ and coroots $\alpha^{\vee} \in \mathfrak{k} \otimes \mathbb{C}$. The list of $m_{k}$ is standard (see [17, $\left.\S 1.5\right]$ or [2, Chap. VIII, $\S 13$ $(\mathrm{VI})])$. 
(i) When $D=\mathbb{R}, K=\mathrm{SO}_{d}(\mathbb{R})$ and $\mathfrak{k}=\mathfrak{s o}_{d}(\mathbb{R})$ is the Lie algebra of antisymmetric matrices (which all have trace 0 ), and we have $\mathfrak{k}_{\mathbb{C}} \cong \mathfrak{s o}_{d}(\mathbb{C})$. We choose $\mathfrak{t} \subset \mathfrak{k}$ to be the space of matrices that are block-diagonal with $2 \times 2$ blocks of the form $\left(\begin{array}{cc}0 & \theta_{k} \\ -\theta_{k} & 0\end{array}\right)$ for $k=1, \ldots, r$. Then $\mathfrak{t}_{\mathbb{Z}} \subset \mathfrak{t}$ is the lattice of elements with $\theta_{k} \in \mathbb{Z}$ for all $k$. We will also write $\theta_{k}$ the corresponding linear form on $\mathfrak{t}$. For $k<\ell \leq r$ and $M \in \mathrm{M}_{2}(\mathbb{R})$, let $R_{k, \ell}(M)$ be the block matrix $\left(\begin{array}{cc}0 & M \\ -{ }^{t} M & 0\end{array}\right)$ embedded in the $(2 k-1,2 k, 2 \ell-1,2 \ell)$-th block of a $d \times d$ matrix. For $k \leq r$ and $v \in \mathbb{R}^{2}$ a column vector, let $R_{k}(v)$ be the block matrix $\left(\begin{array}{cc}0 & v \\ -{ }^{t} v & 0\end{array}\right)$, embedded in the $(2 k-1,2 k, d)$-th block of a $d \times d$ matrix. For $k \leq r$, let $F_{k} \in \mathfrak{t}$ be the matrix with $\theta_{k}=1$ and all other coefficients 0 . Then the roots of $\mathfrak{k}$ with respect to $\mathfrak{t}$ are:

(a) For $1 \leq k<\ell \leq r$, the $\alpha= \pm\left(\theta_{k}-\theta_{\ell}\right) i$, with corresponding

$$
X_{\alpha}=R_{k, \ell}\left(\begin{array}{ll}
1 & 0 \\
0 & 1
\end{array}\right) \pm R_{k, \ell}\left(\begin{array}{cc}
0 & 1 \\
-1 & 0
\end{array}\right) \otimes i \in \mathfrak{k} \otimes \mathbb{C}
$$

and coroots $\alpha^{\vee}= \pm\left(F_{k}-F_{\ell}\right) \otimes i \in \mathfrak{t} \otimes \mathbb{C}$.

(b) For $1 \leq k<\ell \leq r$, the $\alpha= \pm\left(\theta_{k}+\theta_{\ell}\right) i$, with corresponding

$$
X_{\alpha}=R_{k, \ell}\left(\begin{array}{cc}
1 & 0 \\
0 & -1
\end{array}\right) \pm R_{k, \ell}\left(\begin{array}{ll}
0 & 1 \\
1 & 0
\end{array}\right) \otimes i
$$

and coroots $\alpha^{\vee}= \pm\left(F_{k}+F_{\ell}\right) \otimes i$.

(c) If $d$ is odd, for $1 \leq k \leq r$, the $\alpha= \pm \theta_{k} i$, with corresponding

$$
X_{\alpha}=R_{k}\left(\begin{array}{l}
1 \\
0
\end{array}\right) \pm R_{k}\left(\begin{array}{l}
0 \\
1
\end{array}\right) \otimes i
$$

and coroots $\alpha^{\vee}= \pm 2 F_{k} \otimes i$.

(ii) When $D=\mathbb{C}, K=\mathrm{SU}_{d}(\mathbb{C})$ and $\mathfrak{k}=\mathfrak{s u}_{d}(\mathbb{C})$ is the Lie algebra of anti-Hermitian matrices with trace 0 , and we have $\mathfrak{k} \otimes \mathbb{C} \cong \mathfrak{s l}_{d}(\mathbb{C})$. We choose $\mathfrak{t} \subset \mathfrak{k}$ to be the space of diagonal matrices with coefficients $i \theta_{k}$ where $\theta_{k} \in \mathbb{R}$ and $\sum_{k=1}^{d} \theta_{k}=0$. Then $\mathfrak{t}_{\mathbb{Z}} \subset \mathfrak{t}$ is the lattice of elements with $\theta_{k} \in \mathbb{Z}$ for all $k$. We will again write $\theta_{k}$ the corresponding linear form on $\mathfrak{t}$. For $k<\ell \leq r$ and $z \in \mathbb{C}$, let $R_{k, \ell}(z)$ be the matrix $\left(\begin{array}{cc}0 & z \\ -\bar{z} & 0\end{array}\right)$, embedded in the $(k, \ell)$-th block of a $d \times d$ matrix. For $k \leq d$, let $F_{k}$ be the matrix with $\theta_{k}=1$ and all other coefficients 0 . Then the roots of $\mathfrak{k}$ are, for $1 \leq k<\ell \leq d$, the $\alpha= \pm\left(\theta_{k}-\theta_{\ell}\right) i$, with corresponding

$$
X_{\alpha}=R_{k, \ell}(1) \pm R_{k, \ell}(i) \otimes i
$$

and coroots $\alpha^{\vee}= \pm\left(F_{k}-F_{\ell}\right) \otimes i$.

(iii) When $D=\mathbb{H}, K=\mathrm{SU}_{d}(\mathbb{H})$ and $\mathfrak{k}=\mathfrak{s u}_{d}(\mathbb{H})$ is the Lie algebra of quaternionanti-Hermitian matrices (which automatically have reduced trace 0 ), and we have $\mathfrak{k} \otimes \mathbb{C} \cong \mathfrak{s p}_{2 d}(\mathbb{C})$. We choose $\mathfrak{t} \subset \mathfrak{k}$ to be the space of diagonal matrices with coefficients $i \theta_{k}$ where $\theta_{k} \in \mathbb{R}$. Then $\mathfrak{t}_{\mathbb{Z}} \subset \mathfrak{t}$ is the lattice of elements with $\theta_{k} \in \mathbb{Z}$ for all $k$. We will again write $\theta_{k}$ the corresponding linear form on $\mathfrak{t}$. For $k<\ell \leq r$ and $w \in \mathbb{H}$, let $R_{k, \ell}(w)$ be the matrix $\left(\begin{array}{cc}0 & w \\ -\bar{w} & 0\end{array}\right)$, embedded in 
the $(k, \ell)$-th block of a $d \times d$ matrix. For $k \leq r$, let $F_{k}$ be the matrix with $\theta_{k}=1$ and all other coefficients 0 . Then the roots of $\mathfrak{k}$ are

(a) For $1 \leq k<\ell \leq d$, the $\alpha= \pm\left(\theta_{k}-\theta_{\ell}\right) i$, with corresponding

$$
X_{\alpha}=R_{k, \ell}(1) \pm R_{k, \ell}(i) \otimes i
$$

and coroots $\alpha^{\vee}= \pm\left(F_{k}-F_{\ell}\right) \otimes i$.

(b) For $1 \leq k<\ell \leq d$, the $\alpha= \pm\left(\theta_{k}+\theta_{\ell}\right) i$, with corresponding

$$
X_{\alpha}=R_{k, \ell}(j) \pm R_{k, \ell}(i j) \otimes i
$$

and coroots $\alpha^{\vee}= \pm\left(F_{k}+F_{\ell}\right) \otimes i$.

(c) For $1 \leq k \leq d$, the $\alpha= \pm 2 \theta_{k} i$, with corresponding

$$
X_{\alpha}=j F_{k} \pm i j F_{k} \otimes i
$$

and coroots $\alpha^{\vee}= \pm F_{k} \otimes i$.

Computing $\mu\left(\mathfrak{t} / \mathfrak{t}_{\mathbb{Z}}\right)$ and the norms of the coroots in each case gives the result from Macdonald's formula: in his notation we have

$$
\lambda=\mu\left(\mathfrak{t} / \mathfrak{t}_{\mathbb{Z}}\right) \prod_{\alpha}\left\|\alpha^{\vee}\right\|,
$$

and we finally let $\kappa=2^{r} \lambda$.

Corollary 12. - Let $K$ be as above. We have

$$
\log \mu(K)=-\frac{n}{4}(e d)^{2} \log d+O\left(d^{2}\right) .
$$

Proof. - Use Proposition 11 and $\sum_{k=1}^{r} k \log k=\frac{r^{2}}{2} \log r+O\left(r^{2}\right)$.

4.3. Volume of a ball. - In order to find a lower bound for the volume of certain balls $\mathrm{B}(t)$, we will need to compute a lower bound for an integral of the form

$$
\int \prod_{i<j} \sinh \left(a_{i}-a_{j}\right)^{m} d a_{i}
$$

where the integral is over the $\left(a_{i}\right)_{i}$ with $\left|a_{i}\right| \leq t$ and $\sum_{i} a_{i}=0$. The domain for the $a_{i}$ is the intersection of a hypercube with the hyperplane $\sum a_{i}=0$, i.e. a simplex. On the other hand, the integrand is small when some $\left|a_{i}-a_{j}\right|$ is small, that is when $\left(a_{i}\right)$ is close to one of the hyperplanes $a_{i}=a_{j}$. To find a lower bound, we will restrict to a subset of the simplex that is far from those hyperplanes, and where the $a_{i}$ vary independently, so that we can compute the integral. Moreover, the integrand increases exponentially, so the size of the subset does not contribute significantly to the value of the integral, while the values of the integrand do; so we need a subset where many of the $a_{i}$ are close to $t$. This is achieved using the following technical lemma.

Lemma 13. - Let $k \geq 1$ be an integer. Then there exists $k$ intervals $\left[\alpha_{i}, \beta_{i}\right]$ such that for all $a_{i} \in\left[\alpha_{i}, \beta_{i}\right]$ we have:

(1) $\left|a_{i}\right| \leq 1$;

(2) $\left|\sum_{j=1}^{k} a_{j}\right| \leq 1$;

(3) $\beta_{i}-\alpha_{i} \geq \frac{1}{4(k+1)^{2}}$;

(4) $\left|a_{i}-a_{j}\right| \geq \frac{1}{4(k+1)^{2}}$

(5) $\left|a_{i}+\sum_{j=1}^{k} a_{j}\right| \geq \frac{1}{4(k+1)^{2}}$; 
(6) $\left|\left\{j: a_{j} \geq \frac{1}{4}\right\}\right| \geq \frac{k+1}{5}$.

Proof. - Let $c_{1}, \ldots, c_{k}$ be defined by $c_{i}=\frac{2 i}{k+1}-1$, except when $k$ is odd, where $c_{\frac{k+1}{2}}=$ $\frac{1}{k+1}$. Let $\alpha_{i}=c_{i}-\frac{1}{8(k+1)^{2}}$ and $\beta_{i}=c_{i}+\frac{1}{8(k+1)^{2}}$. We claim that they satisfy the required properties.

(1) In absolute value, the extremal points of the intervals are $\frac{k-1}{k+1}+\frac{1}{8(k+1)^{2}} \leq 1$.

(2) The sum $\sum_{j=1}^{k} c_{j}$ is 0 if $k$ is even, and $\frac{1}{k+1}$ if $k$ is odd. The required inequalities become $\left|\frac{k}{8(k+1)^{2}}\right| \leq 1$ and $\left|\frac{1}{k+1}+\frac{k}{8(k+1)^{2}}\right| \leq 1$, which are true.

(3) By definition we have $\beta_{i}-\alpha_{i}=\frac{1}{4(k+1)^{2}}$.

(4) The minimum separation between the centres $c_{i}$ is $\frac{1}{k+1}$, and we have $\frac{1}{k+1}-\frac{1}{4(k+1)^{2}} \geq$ $\frac{1}{4(k+1)^{2}}$.

(5) The minimum separation between a $c_{i}$ and $-\sum_{j=1}^{k} c_{j}$ is at least $\frac{1}{k+1}$, and we have $\frac{1}{k+1}-$ $\frac{1}{8(k+1)^{2}}-\frac{k}{8(k+1)^{2}} \geq \frac{1}{4(k+1)^{2}}$.

(6) If $k \leq 4$ it is obvious, and when $k \geq 5$ we have $\alpha_{i} \geq \frac{1}{4}$ if and only if $i \geq \frac{k+1}{8}\left(5+\frac{1}{2(k+1)^{2}}\right)$, and the number of such $i$ is at least $\frac{k+1}{5}$.

We can now give a lower bound for the volume of a certain ball $\mathrm{B}(t) \subset G$ with the above Haar measure $d \mu$.

Proposition 14. - Let $t \geq 1$, and let $\mathrm{B}(t)=\{g \in G \mid \rho(g) \leq t\}$. Assume $d \geq 2$. Then

$$
\log \mu(\mathrm{B}(t)) \geq-\frac{3 n(d e)^{2}}{2} \log d+\frac{n(d e)^{2}}{200} t+O\left(d^{2}\right) .
$$

Proof. - We apply Proposition [10] with $f$ the indicator function of $\mathrm{B}(t)$. The formula reads

$$
\mu(\mathrm{B}(t))=\frac{(n e)^{\frac{d-1}{2}} \sqrt{d}}{\mu\left(\mathrm{Z}_{K}(A)\right)} \mu(K)^{2} I, \text { where } I=\int_{a_{i}} \prod_{i<j} \sinh \left(a_{i}-a_{j}\right)^{n e^{2}} d a_{i},
$$

where the integral is over the $a_{i} \in[-t, t]$ with $\left(a_{i}\right)_{i}$ decreasing and $\sum_{i} a_{i}=0$. To compute a lower bound for the integral, we apply Lemma 13 to $k=d-1$. We have intervals $\left[\alpha_{i}, \beta_{i}\right]$, and after reordering the intervals and scaling them by $t$, we obtain that

$$
I \geq \int \prod_{i<j} \sinh \left(a_{i}-a_{j}\right)^{n e^{2}} d a_{i}
$$

where for $i<d, a_{i}$ ranges over some interval $\left[t \alpha_{\sigma(i)}, t \beta_{\sigma(i)}\right]$, and $a_{d}=-\sum_{i=1}^{d-1} a_{i}$. Since $x \mapsto \sinh (x) \exp (-x)=\frac{1-\exp (-2 x)}{2}$ is increasing, for all $x \geq \frac{t}{4 d^{2}}$ we have

$$
\sinh (x) \geq \frac{1-\exp \left(-t / 2 d^{2}\right)}{2} \exp (x) \geq \frac{1-\exp \left(-1 / 2 d^{2}\right)}{2} \exp (x) \geq \frac{1}{4 d^{2}} \exp (x) .
$$

We get

$$
I \geq\left(\frac{1}{4 d^{2}}\right)^{n e^{2} d(d-1) / 2} \int \prod_{i<j} \exp \left(a_{i}-a_{j}\right)^{n e^{2}} d a_{i}=\left(\frac{1}{4 d^{2}}\right)^{n e^{2} d(d-1) / 2} \int \exp \left(\sum_{i<j}\left(a_{i}-a_{j}\right)\right)^{n e^{2}} d a_{i} .
$$


We compute the term $\beta$ that appears in the exponential. For all $\left(a_{i}\right)$ such that $\sum_{i=1}^{d} a_{i}=0$, we have

$$
\begin{aligned}
\beta & =\sum_{1 \leq i<j \leq d}\left(a_{i}-a_{j}\right)=\sum_{i<j} a_{i}-\sum_{i<j} a_{j} \\
& =\sum_{i}(d-i) a_{i}-\sum_{j}(j-1) a_{j}=\sum_{i=1}^{d}(d+1-2 i) a_{i} \\
& =-2 \sum_{i=1}^{d} i a_{i} .
\end{aligned}
$$

Now since $a_{d}=-\sum_{i=1}^{d-1} a_{i}$, we have $\beta=2 \sum_{i=1}^{d-1}(d-i) a_{i}$. This gives

$$
I \geq(2 d)^{-d(d-1) n e^{2}} \int \exp \left(2 n e^{2} \sum_{i=1}^{d-1}(d-i) a_{i}\right) d a_{i} .
$$

By properties (3) and (6) of Lemma 13, we obtain

$$
I \geq(2 d)^{-d(d-1) n e^{2}} \exp \left(2 n e^{2} \sum_{i=1}^{\lfloor d / 5\rfloor} i \frac{t}{4}\right)\left(\frac{1}{4 d^{2}}\right)^{(d-1) n e^{2}} \geq(2 d)^{-(d-1)(d+2) n e^{2}} \exp \left(\frac{d^{2} n e^{2}}{200} t\right) .
$$

In particular,

$$
\log I \geq-n(d e)^{2} \log d+\frac{n(d e)^{2}}{200} t+O\left(d^{2}\right) .
$$

We conclude by using Corollary 12 and Lemma 9.

\section{Multiplicative construction}

We consider the following arithmetic group code. Let $F$ be a number field of degree $n$ over $\mathbb{Q}$, and let $A$ be a central division algebra of degree $d \geq 2$ over $F$. If $d=2$, assume that $A$ is unramified at all real places. Let $\mathcal{O}$ be a maximal order in $A$. We let $\mathbb{G}$ be the algebraic group defined by the reduced norm 1 subgroup $A^{1} \subset A^{\times}$, and

$$
\Gamma=\mathcal{O}^{1}=\{x \in \mathcal{O} \mid \operatorname{nrd}(x)=1\} .
$$

Let $S$ be a set of prime ideals of $\mathbb{Z}_{F}$ that are unramified in $A$ and such that for all $\mathfrak{p} \in S$, the residue field $\mathbb{Z}_{F} / \mathfrak{p}$ is isomorphic to a common finite field $\mathbb{F}_{q_{0}}$. For all $\mathfrak{p} \in S$, we fix an isomorphism $\iota_{\mathfrak{p}}: \mathcal{O} / \mathfrak{p} \mathcal{O} \cong \mathrm{M}_{d}\left(\mathbb{F}_{q_{0}}\right)$.

Let $\mathcal{A}=\mathrm{M}_{d}\left(\mathbb{F}_{q_{0}}\right)$ so that $q=q_{0}^{d}, s=|S|$ and $N=d s$, and define $\Theta: \Gamma \rightarrow \mathcal{A}^{s}$ to be the map sending $\gamma \in \Gamma$ to the word formed by the $\iota_{\mathfrak{p}}(\gamma)$ for $\mathfrak{p} \in S$.

Let us write $n=r_{1}+2 r_{2}$ and $r_{1}=u+r$, where $\left(r_{1}, r_{2}\right)$ is the signature of $F, u$ is the number of real places $\sigma$ that are unramified in $A$, and $r$ is the number of real places $\sigma$ that ramify in $A$. Let

$$
G=\prod_{\sigma \in \mathbb{P}_{\infty}} \mathrm{SL}_{d_{\sigma}}\left(D_{\sigma}\right) \cong \mathrm{SL}_{d}(\mathbb{R})^{u} \times \mathrm{SL}_{d / 2}(\mathbb{H})^{r} \times \mathrm{SL}_{d}(\mathbb{C})^{r_{2}}
$$

Following the notations of Section 4 , we define $\rho: G \rightarrow \mathbb{R}_{\geq 0}$ componentwise: for all $g=$ $\left(g_{\sigma}\right)_{\sigma \in \mathbb{P}_{\infty}} \in G$, let

$$
\rho(g)=\max _{\sigma \in \mathbb{P}_{\infty}} \rho\left(g_{\sigma}\right) .
$$


For $t>0$, we define the following compact subset $\mathrm{B}(t) \subset G$ :

$$
\mathrm{B}(t)=\{g \in G \mid \rho(g) \leq t\} .
$$

Note that for all $g, h \in \mathrm{B}(t)$, we have $h^{-1} \in \mathrm{B}(t)$ and $\rho\left(h^{-1} g\right) \leq 2 t$, by Corollary 7 . Let $\mathcal{C}$ be the code attached to $(\mathrm{B}(t), \Gamma, \Theta)$ as in Section 2. The goal of this section is to analyse the code $\mathcal{C}$, and to obtain aymptotically good families of codes from this construction.

5.1. Minimal distance. — Let us start with the following lemma:

Lemma 15. - Let $A$ be a central simple algebra of degree d over a number field $F$, and let $\mathcal{O}$ be an order in $A$. Let $\mathfrak{p}$ be a prime ideal of $\mathbb{Z}_{F}$ such that there is an isomorphism $\iota_{\mathfrak{p}}: \mathcal{O} / \mathfrak{p} \mathcal{O} \cong \mathrm{M}_{d}\left(\mathbb{F}_{q}\right)$. Let $x \in \mathcal{O}$, and let $r$ be the rank of the matrix $\iota_{\mathfrak{p}}(x)$. Then $|\mathcal{O} /(\mathfrak{p O}+x \mathcal{O})|=q^{d(d-r)}$.

Proof. - Let $m=\iota_{\mathfrak{p}}(x)$. We have $\mathcal{O} /(\mathfrak{p O}+x \mathcal{O}) \cong \mathrm{M}_{d}\left(\mathbb{F}_{q}\right) /\left(m \cdot \mathrm{M}_{d}\left(\mathbb{F}_{q}\right)\right)$. Since $\operatorname{dim}_{\mathbb{F}_{q}}(m$. $\left.\mathbb{F}_{q}^{d}\right)=r$ by definition, we have $\operatorname{dim}_{\mathbb{F}_{q}}\left(m \cdot \mathrm{M}_{d}\left(\mathbb{F}_{q}\right)\right)=d r$, and therefore $\operatorname{dim}_{\mathbb{F}_{q}} \mathrm{M}_{d}\left(\mathbb{F}_{q}\right) /(m$. $\left.\mathrm{M}_{d}\left(\mathbb{F}_{q}\right)\right)=d(d-r)$, proving the result.

Concerning the minimum sum-rank distance $d_{R}(\mathcal{C})$ of the code $\mathcal{C}$, we obtain:

Proposition 16. - We have

$$
d_{R}(\mathcal{C}) \geq N-n d^{2} \frac{\log 2}{\log q}-\frac{2 n d^{2} t}{\log q}
$$

Proof. - Let $x \neq y$ be elements of $\Gamma \cap c \mathrm{~B}(t)$ such that $d_{R}(\Theta(x), \Theta(y))=d_{R}(\mathcal{C})$. Note that since $y \in \Gamma$, the matrix $\iota_{\mathfrak{p}}(y)$ has determinant 1 and is invertible with inverse $\iota_{\mathfrak{p}}(y)^{-1}=$ $\iota_{\mathfrak{p}}\left(y^{-1}\right)$. Let $z=y^{-1} x-1 \in \mathcal{O}$; the element $z$ is nonzero and therefore $\mathrm{N}(z) \neq 0$ since $A$ is a division algebra. For each $\mathfrak{p} \in S$, let $r_{\mathfrak{p}}$ be the rank of $\iota_{\mathfrak{p}}(z)$. Since multiplying a matrix by the invertible matrix $\iota_{\mathfrak{p}}(y)^{-1}$ on the left does not change the rank, we get $\sum_{\mathfrak{p} \in S} r_{\mathfrak{p}}=d_{R}(\mathcal{C})$. Moreover, for all $\mathfrak{p} \in S$ we have $|\mathcal{O} /(\mathfrak{p O}+z \mathcal{O})| \geq q_{0}^{d\left(d-r_{\mathfrak{p}}\right)}$ by Lemma 15. We obtain

$$
\mathrm{N}(z)=|\mathcal{O} / z \mathcal{O}| \geq \prod_{\mathfrak{p} \in S}|\mathcal{O} /(\mathfrak{p O}+z \mathcal{O})| \geq \prod_{\mathfrak{p} \in S} q_{0}^{d\left(d-r_{\mathfrak{p}}\right)}=q_{0}^{d\left(N-d_{R}(\mathcal{C})\right)}=q^{N-d_{R}(\mathcal{C})} .
$$

On the other hand, let us write $x=c x_{0}$ and $y=c y_{0}$, with $x_{0}, y_{0} \in \mathrm{B}(t)$ and where $c \in G$ is as in Section 2, Since $\rho\left(y^{-1} x\right)=\rho\left(y_{0}^{-1} x_{0}\right) \leq 2 t$, by Corollary 8 we obtain

$$
\mathrm{N}(z)=\prod_{\sigma: F \hookrightarrow \mathbb{C}}|\sigma(\operatorname{nrd}(z))|^{d} \leq 2^{n d^{2}} \exp \left(n d^{2} \rho\left(y^{-1} x\right)\right) \leq 2^{n d^{2}} \exp \left(2 n d^{2} t\right) .
$$

Taking logarithms and dividing by $\log q$ gives the result.

As consequence, we have:

Corollary 17. - Suppose that $t>0$ is such that $2 t \leq \frac{N \log q}{n d^{2}}-\log 2$. Then $\left.\Theta\right|_{\Gamma \cap c \mathrm{~B}(t)}$ is injective. 
5.2. Number of codewords. - Recall that $G=\prod_{\sigma \in \mathbb{P}_{\infty}} \mathrm{SL}_{d_{\sigma}}\left(D_{\sigma}\right)$. Then $G$ inherits the product topology and the product measure $\otimes_{\sigma} d \mu_{\sigma}$, where the volume form $d \mu_{\sigma}$ is normalised as in Section 4.2. We start with Prasad's formula for the volume $G / \mathcal{O}^{1}$.

Proposition 18. - We have

$$
\mu\left(G / \mathcal{O}^{1}\right)=d^{\frac{n}{2}}\left(\frac{\Delta_{A}}{\Delta_{F}}\right)^{1 / 2} \prod_{j=2}^{d} \zeta_{F}(j) \cdot \Phi
$$

where

$$
\Phi=\prod_{\mathfrak{p}} \prod_{0<i<d, e_{\mathfrak{p}} \nmid i}\left(1-N(\mathfrak{p})^{-i}\right) .
$$

Proof. - We use Prasad's formula [17, Theorem 3.7] where the normalisation of the volume is defined as follows. On each factor $\mathfrak{g}_{\sigma}=\mathfrak{s l}_{d_{\sigma}}\left(D_{\sigma}\right)$, the choice of a volume form determines one on $\mathfrak{g}_{\sigma} \otimes_{\mathbb{R}} \mathbb{C}$. Prasad chooses the volume form that gives volume 1 to a maximal compact subgroup $K^{\prime} \cong \mathrm{SU}_{d}(\mathbb{C})^{n}$ of $\mathbb{G}(\mathbb{C})=\left(A \otimes_{\mathbb{R}} \mathbb{C}\right)^{1}$. For this normalisation, Prasad's formula yields

$$
\mu_{\text {Pras }}\left(G / \mathcal{O}^{1}\right)=\left(\frac{\Delta_{A}}{\Delta_{F}}\right)^{1 / 2}\left(\prod_{k=1}^{d-1} \frac{k !}{(2 \pi)^{k+1}}\right)^{n} \prod_{j=2}^{d} \zeta_{F}(j) \cdot \Phi
$$

(for details, the reader can refer to [14, Theorem 2.4.1.10]). To relate our normalisation to the one of Prasad, we relate the norm $\|\cdot\|$ on $\mathfrak{s l}_{d_{\sigma}}\left(D_{\sigma}\right) \otimes_{\mathbb{R}} \mathbb{C} \cong \mathfrak{s l}_{d}(\mathbb{C})^{\left[F_{\sigma}: \mathbb{R}\right]}$ that we defined previously, to the norm $\|\cdot\|_{\mathbb{C}}$ on the same Lie algebra, induced by the one on $\mathfrak{s l}_{d_{\sigma}}\left(D_{\sigma}\right)$ : we find $\|\cdot\|_{\mathbb{C}}=\frac{1}{\sqrt{2}}\|\cdot\|$. Denoting $\mu_{\mathbb{C}}$ the measure induced by the metric $\|\cdot\|_{\mathbb{C}}$, we have

$$
\mu_{\mathbb{C}}\left(K^{\prime}\right)=2^{-\frac{n\left(d^{2}-1\right)}{2}} \mu\left(\mathrm{SU}_{d}(\mathbb{C})\right)^{n}=2^{-\frac{n\left(d^{2}-1\right)}{2}}\left(2^{d^{2}+d / 2-3 / 2} \sqrt{d} \prod_{k=1}^{d-1} \frac{\pi^{k+1}}{k !}\right)^{n}
$$

by Proposition 11(iii). Finally, we get the formula since

$$
\mu\left(G / \mathcal{O}^{1}\right)=\mu_{\operatorname{Pras}}\left(G / \mathcal{O}^{1}\right) \mu_{\mathbb{C}}\left(K^{\prime}\right) .
$$

Corollary 19. - We have

$$
\log \mu\left(G / \mathcal{O}^{1}\right) \leq \frac{1}{2} \log \left(\frac{\Delta_{A}}{\Delta_{F}}\right)+O(n \log d) .
$$

Proof. - It follows from $\Phi \leq 1$ and $\zeta_{F}(j) \leq \zeta(j)^{n} \leq\left(1+O\left(2^{-j}\right)\right)^{n}$ for $j \geq 2$.

This estimate allows us to obtain the following bound on the rate of $\mathcal{C}$ :

Proposition 20. - Assume that $s=|S|=n$. For all $t \geq 1$ as in Corollary 17, we have

$$
\frac{1}{N} \log |\mathcal{C}| \geq \frac{d}{200} t-\frac{d^{2}-1}{2 d} \log \operatorname{rd}_{F}-\frac{1}{2 n} \log \mathrm{N}\left(\delta_{A}\right)-\frac{3 d}{2} \log d+O(d) .
$$

Proof. - We have $N=d n$, and by Lemma 1, $|\mathcal{C}| \geq \mu(\mathrm{B}(t)) / \mu\left(G / \mathcal{O}^{1}\right)$. Since $\mathrm{B}(t)$ is a product of balls on each of the factors, Proposition 14, together with Corollary 19] and the relation $\Delta_{A}=N\left(\delta_{A}\right)^{d} \Delta_{F}^{d^{2}}$, gives the result. 
5.3. Analysis of the code. - Let us start with the existence of unramified towers of number fields with splitting conditions.

Proposition 21. - There exist an integer $M_{0}$ and a real number $C>0$ such that for all primes $p$ satisfying $\left(\frac{M_{0}}{p}\right)=1$, there exists a sequence of number fields $\left(F_{k}\right)_{k}$ such that: $(i)\left[F_{k}: \mathbb{Q}\right] \rightarrow \infty$, (ii) the prime $p$ splits totally in $F_{k} / \mathbb{Q}$, and $(i i i) \operatorname{rd}_{F_{k}} \leq C$.

Proof. - This follows from well-known methods in the study of towers of bounded root discriminant, using a tower above a quadratic field. The reader may refer to [11, Section 5].

We now prove the main result of this work.

Theorem 22. - For all $d \geq 2$, there exists a family of asymptotically good number field codes for the sum-rank distance, each obtained from the group of units of reduced norm 1 in a maximal order in a division algebra of degree $d$, over a fixed alphabet $\mathrm{M}_{d}\left(\mathbb{F}_{p}\right)$, where $\log p=c \log d+O(\log \log d)$ and $c>0$ is a constant.

Proof. - Let $d \geq 2$. We pick a family of number fields $F_{k}$ with $\operatorname{rd}_{F_{k}} \leq C$ as in Proposition 21, leaving $p \geq 5$ to be chosen later. Fix $F_{k}$ and let $n=\left[F_{k}: \mathbb{Q}\right]$. We choose $A$ a central division algebra of degree $d$ over $F_{k}$ ramified exactly at one prime $\mathfrak{p}_{2}$ above 2 and one prime $\mathfrak{p}_{3}$ above 3 ; by Class Field Theory such an algebra does exist (see for instance $[\mathbf{1 8}$, Section 32, especially (32.13)] and the references therein). This implies $\mathrm{N}\left(\delta_{A}\right)^{\frac{1}{n d}} \leq 6$. We choose $S$ to be the set of primes of $F_{k}$ above $p$, so that $q_{0}=p, q=p^{d}, s=n=\left[\mathbb{F}_{k}: \mathbb{Q}\right]$ and $N=n d$. Let $\mathcal{C}$ be the unit group code constructed from $A$. The quantity of Proposition 20

$$
\frac{d}{200} t-\frac{d^{2}-1}{2 d} \log \mathrm{rd}_{F}-\frac{1}{2 n} \log \mathrm{N}\left(\delta_{A}\right)-\frac{3 d}{2} \log d+O(d)
$$

can be written as

$$
\frac{d}{200} t-\frac{3 d}{2} \log d+f(d) \text { where } f(d)=O(d)
$$

We pick $t \geq 1$ minimal such that $\frac{d}{200} t-\frac{3 d}{2} \log d+f(d) \geq 1$, so that $t=300 \log d+O(1)$. By Proposition 16 we have

$$
\frac{d_{R}(\mathcal{C})}{N} \geq 1-\frac{\log 2}{\log p}-\frac{2 t}{\log p}
$$

We pick $p$ such that $\log p \geq \frac{1}{1-d^{-1}}(\log 2+2 t)$, so that $\frac{d_{R}(\mathcal{C})}{N} \geq \frac{1}{d}$. By Proposition 20 and by the choice of $t$, we have

$$
\frac{1}{N} \log |\mathcal{C}| \geq 1
$$

Since any $p \geq 5$ such that $\left(\frac{M_{0}}{p}\right)=1$ can be used, by the Dirichlet arithmetic progression theorem, there exists such $p$ with $\log p=2 t+O(\log t)$, i.e. $\log p=600 \log d+O(\log \log d)$.

Remark 23. - The main contributions come from the volume of $K$ and from the exponential growth rate of the volume of $\mathrm{B}(t)$. We obtain $c=600$ as an admissible value. We did not try to optimise this constant. It would be interesting to find families of unit group codes with a better asymptotic behaviour of $\log p$ as $d \rightarrow \infty$. 
5.4. Quaternion case. - In this section, $A$ is a quaternion algebra, i.e. $d=2$. First, it is easy to give a closed formula for the volume of $\mathrm{B}(t)$.

Proposition 24. - We have

$$
\mu(\mathrm{B}(t))=2^{\frac{3}{2} u+\frac{5}{2} r+4 r_{2}} \pi^{2 r_{1}+3 r_{2}}(\cosh (2 t)-1)^{u}(\sinh (4 t)-4 t)^{r_{2}} .
$$

Proof. - We compute the volume on each factor.

By Proposition 11 , we have $\mu\left(\mathrm{SO}_{2}(\mathbb{R})\right)=2 \sqrt{2} \pi, \mu\left(\mathrm{SU}_{2}(\mathbb{C})\right)=16 \pi^{2}$ and $\mu\left(\mathrm{SL}_{1}(\mathbb{H})\right)=$ $4 \sqrt{2} \pi^{2}$. In the $\mathrm{SL}_{2}(\mathbb{R})$ case, the integral to compute is

$$
\int_{0}^{t} \sinh (2 a) d a=\frac{1}{2}(\cosh (2 t)-1) .
$$

In the $\mathrm{SL}_{2}(\mathbb{C})$ case, the integral involved is

$$
\int_{0}^{t} \sinh (2 a)^{2} d a=\frac{1}{8}(\sinh (4 t)-4 t) .
$$

Putting these together gives the result.

In the quaternion case, Prasad's formula becomes:

Proposition 25. - We have

$$
\mu\left(G / \mathcal{O}^{1}\right)=2^{n / 2}\left(\Delta_{F}\right)^{\frac{3}{2}} \zeta_{F}(2) \prod_{\mathfrak{p} \mid \delta_{A}}(N(\mathfrak{p})-1)
$$

To conclude this section, let us give an explicit example. Let $F=\mathbb{Q}(\cos (2 \pi / 11), \sqrt{2}, \sqrt{-23})$ (from [12]). The 2-class group of $F$ is isomorphic to $(\mathbb{Z} / 2 \mathbb{Z})^{9}$. Let $F_{1}$ be the 2-Hilbert class field of $F$. Let $p$ be a prime number such that every prime ideal above $p$ in $F$ splits completely in $F_{1} / F$; take a such prime ideal $\mathfrak{p}$. Then there exists an unramified infinite extension $L / F$ such that $\mathfrak{p}$ splits completely in $L$ (see [11, Example 9]). Let $F_{1} \subset F_{k} \subset L$ be an intermediate field, of degree $n$ over $\mathbb{Q}$. By construction there exists $n / 20$ primes of $F_{k}$ above $\mathfrak{p}$ of residue degree $f_{p}$, where $f_{p} g_{p}=20$. Let $A$ be the quaternion algebra over $F_{k}$ ramified at exactly two of these primes, and let $S$ be the set of the remaining ones, which has size $s=\frac{n}{20}-2 ; N=d s$. Let $\mathcal{C}$ be the unit group code constructed from $A$. Then

$$
\frac{\mu(\mathrm{B}(t))}{\mu\left(G / \mathcal{O}^{1}\right)}=\frac{\left(2^{2} \pi^{3 / 2}(\sinh (4 t)-4 t)^{1 / 2}\right)^{n}}{2^{n / 2} \operatorname{rd}_{F_{k}}^{3 n / 2} \zeta_{F_{k}}(2)\left(p^{f_{p}}-1\right)^{2}},
$$

in particular

$$
\left(\frac{\mu(\mathrm{B}(t))}{\mu\left(G / \mathcal{O}^{1}\right)}\right)^{1 / n} \geq \frac{(2 \pi)^{3 / 2}(\sinh (4 t)-4 t)^{1 / 2}}{\operatorname{rd}_{F}^{3 / 2} \zeta_{F}(2)^{1 / 20}\left(p^{f_{p}}-1\right)^{2 / n}} .
$$

We have $\operatorname{rd}_{F} \approx 92.37$ and $\zeta_{F}(2) \approx 1.02$. For $t=2.2$ we have

$$
\frac{(2 \pi)^{3 / 2}(\sinh (4 t)-4 t)^{1 / 2}}{\operatorname{rd}_{F}^{3 / 2} \zeta_{F}(2)^{1 / 20}}>1,
$$

so this is an admissible value for an asymptotically good code. From the minimal distance formula, we can choose any $p$ such that $q_{0}=p^{f_{p}}$ satisfies

$$
\log q_{0}>\frac{n}{s}(\log 2+2 t)
$$


Asymptotically we can take any $p$ such that $\frac{1}{20} \log q_{0}>5.09$, i.e. $p^{f_{p} / 20} \geq 163$ and therefore $q=p^{d f_{p}} \geq 163^{40}$.

\section{Complement: the additive construction}

6.1. The construction (following [11]). - Let us recall the additive construction. Let $F$ be a number field of degree $n$ over $\mathbb{Q}$, and let $A$ be a central division algebra of degree $d$ over $F$. Consider the locally compact group $G=\prod_{\sigma \in \mathbb{P}_{\infty}} \mathrm{M}_{d_{\sigma}}\left(D_{\sigma}\right)$, equipped with

- the Euclidean norm $\mathrm{T}_{2}(g)=\sum_{\sigma \in \mathbb{P}_{\infty}} n_{\sigma}\|\sigma(g)\|_{2}^{2}$, where $\left\|\left(m_{i, j}\right)\right\|_{2}=\sqrt{\sum_{i, j} e_{\sigma}\left|m_{i, j}\right|^{2}}$;

- the Lebesque measure $d \mu$ relative to an orthonormal basis of $G$ with respect to $\mathrm{T}_{2}$.

Let $\mathbb{G}=A$ be the algebraic additive group, and $\Gamma=\mathcal{O}$, where $\mathcal{O}$ is a maximal order of $A$. As for the multiplicative group code, let $S$ be a finite set of ideal primes of $\mathbb{Z}_{F}$ that are unramified in $A$ and such that for all $\mathfrak{p} \in S$, the residue field $\mathbb{Z}_{F} / \mathfrak{p}$ is isomorphic to a common finite field $\mathbb{F}_{q_{0}}$. For all $\mathfrak{p} \in S$, we fix an isomorphism $\iota_{\mathfrak{p}}: \mathcal{O} / \mathfrak{p} \mathcal{O} \cong \mathrm{M}_{d}\left(\mathbb{F}_{q_{0}}\right)$.

Let $\mathcal{A}=\mathrm{M}_{d}\left(\mathbb{F}_{q_{0}}\right)$ so that $q=q_{0}^{d}, s=|S|$ and $N=d s$, and define $\Theta: \Gamma \rightarrow \mathcal{A}^{s}$ to be the map sending $\gamma \in \Gamma$ to the word formed by the $\iota_{\mathfrak{p}}(\gamma)$ for $\mathfrak{p} \in S$.

Take the ball $\mathrm{B}(t)=\left\{\left(x_{\sigma}\right)_{\sigma} \in \mathbb{G} \mid\left\|x_{\sigma}\right\|_{2} \leq t\right\}$. Let $\mathcal{C}$ be the code attached to $(\mathrm{B}(t), \Gamma, \Theta)$ as in Section 2.

Proposition 26. - We have

$$
d_{R}(\mathcal{C}) \geq N-d^{2} n \frac{\log (2 t)}{\log q}+\frac{d^{2} n}{2} \cdot \frac{\log d}{\log q} .
$$

Proof. - We follow the proof of Proposition 16. Let $x \neq y$ in $\Gamma \cap(c+\mathrm{B}(t))$ be such that $d_{R}(\Theta(x), \Theta(y))=d_{R}(\mathcal{C})$, where $c \in G$ is as in Section 2. Let $z=x-y \in \mathcal{O}$; the element $z$ is nonzero and therefore $\mathrm{N}(z) \neq 0$. As for the multiplicative case, we obtain $\mathrm{N}(z) \geq q^{N-d_{R}(\mathcal{C})}$. On the other hand, let us write $x=c+x_{0}$ and $y=c+y_{0}$, with $x_{0}, y_{0} \in \mathrm{B}(t)$. As $\|\sigma(x-y)\|_{2} \leq 2 t$, and $\mathrm{T}_{2}(z) \leq n(2 t)^{2}$, then we obtain

$$
\mathrm{N}(z)=\prod_{\sigma: F \hookrightarrow \mathbb{C}}|\sigma(\operatorname{nrd}(z))|^{d} \leq\left(\frac{2 t}{d^{1 / 2}}\right)^{d^{2} n},
$$

thanks to the estimate (see [14, Chapter 2, §3]): $\left|N_{F / \mathbb{Q}} \operatorname{nrd}(x)\right|^{d} \leq\left(\frac{\mathrm{T}_{2}(x)}{d n}\right)^{d^{2} n / 2}$. Taking logarithms and dividing by $\log q$ gives the result.

Concerning the number of codewords, we have:

Proposition 27. - Suppose that $\left.\Theta\right|_{\Gamma \cap(c+\mathrm{B}(t))}$ is injective. Then we have

$$
|\mathcal{C}| \geq \frac{2^{r_{2} d^{2}} \mathbb{V}_{d^{2}}^{r_{1}} \mathbb{V}_{2 d^{2}}^{r_{2}} t^{d^{2} n}}{\sqrt{\Delta_{A}}}
$$

where $\mathbb{V}_{n}$ denotes the volume of the unit ball of the space $\mathbb{R}^{n}$ equipped with the Lebesgue volume form. 
Proof. - Here $\mu(\mathrm{B}(t))=2^{r_{2} d^{2}} \mathbb{V}_{d^{2}}^{r_{1}} \mathbb{V}_{2 d^{2}}^{r_{2}} t^{d^{2} n}$, and $\mu(G / \mathcal{O})=\sqrt{\Delta_{A}}(\dagger)$. Then apply Lemma 1.

Corollary 28. - Suppose that $\left.\Theta\right|_{\Gamma \cap(c+\mathrm{B}(t))}$ is injective. Then we have

$$
\log |\mathcal{C}| \geq n d^{2} \log t-\frac{1}{2} \log \Delta_{A}-n d^{2} \log d+O\left(n d^{2}\right) .
$$

Proof. - Use $\mathbb{V}_{n}=-\frac{n}{2} \log n+O(n)$.

6.2. Asymptotic analysis. - We now follow the case of the multiplicative group of Section 5.3 to obtain the following

Theorem 29. - For all $d \geq 2$, there exists a family of asymptotically good number field codes for the sum-rank distance, each obtained from the additive group of a maximal order in a division algebra of degree $d$, over a fixed alphabet $\mathrm{M}_{d}\left(\mathbb{F}_{p}\right)$, where $\log p=$ $\frac{1}{2} \log d+O(\log \log d)$.

Proof. - Let $d \geq 2$. As in the proof of Theorem 22, we pick a family of number fields $F_{k}$, a prime number $p$, a central division algebra $A$ ramified exactly at one prime $\mathfrak{p}_{2}$ above 2 and at one prime $\mathfrak{p}_{3}$ above 3 , and a set $S$ of primes, so that $q_{0}=p, q=p^{d}, s=n=\left[\mathbb{F}_{k}: \mathbb{Q}\right]$ and $N=n d$. Let $\mathcal{C}$ be the additive group code constructed from $A$ and $S$. Then by Corollary 28 we have

$\frac{1}{N} \log |\mathcal{C}| \geq d \log t-\frac{1}{2 n d} \log \Delta_{A}-d \log d+O(d)=d \log t-d \log d+f(d)$ where $f(d)=O(d)$.

We pick $t \geq 1$ minimal such that $-d \log d+d \log t+f(d) \geq 1$, so that

$$
\log t=\log d+O(1)
$$

By Proposition 26 we have

$$
\frac{d_{R}(\mathcal{C})}{N} \geq 1-\frac{\log 2 t}{\log p}+\frac{\log d}{2 \log p}
$$

We pick $p$ such that $\log p \geq \frac{1}{1-d^{-1}}\left(\frac{1}{2} \log d+O(1)\right)$, so that $\frac{d_{R}(\mathcal{C})}{N} \geq \frac{1}{d}$. By Proposition 27 (and by the choice of $t$ ), we have $\frac{1}{N} \log |\mathcal{C}| \geq 1$. As before, by the Dirichlet arithmetic progression theorem, there exists such $p$ with $\log p=\frac{1}{2} \log d+O(\log \log d)$.

6.3. Codes over finite fields. - In this section, we explain how to construct codes from quaternion orders, with alphabet naturally given as a finite field. The difference with the previous constructions is that the primes in $S$ need to ramify in $A$.

Theorem 30. - Let $M_{0}$ and $C$ as in Proposition 21, and let $\alpha=-\frac{1}{4} \log \frac{\pi^{4}}{24}$. For all prime $p$ such that $\left(\frac{M_{0}}{p}\right)=1$ and $\log p>\log C+\alpha$, there exists a family of asymptotically good codes over $\mathbb{F}_{p^{2}}$, each obtained from the additive group of a maximal order in a quaternion algebra.

†. A factor $2^{-r_{2} d^{2}}$ is missing in [11, Proposition 9], but this does not affect the results of the paper. 
Proof. - Let $F_{k}$ be as in Proposition 21, Let $p$ be a prime number that splits totally in $F_{k} / \mathbb{Q}$, and let $S$ be a maximal subset of primes of $F_{k}$ above $p$ such that $|S|$ is even. Let $A$ be a central division algebra of degree $d$ over $F_{k}$ ramified exactly at each prime ideal $\mathfrak{p}$ of $S$ and with common ramification index $e>1$. Consider the additive codes as in the previous section. Writing $d=$ ef and $\mathfrak{P}$ the maximal two-sided ideal of $\mathcal{O}$ above $\mathfrak{p}$, then there is an isomorphism $\iota_{\mathfrak{p}}: \mathcal{O} / \mathfrak{P} \cong \mathrm{M}_{f}\left(\mathbb{F}_{p^{e}}\right)$, and we use columns $\mathbb{F}_{p^{e}}^{f}$ as codewords via $\iota_{\mathfrak{p}}$. We have $N=f \cdot|S| \geq f \cdot\left(\left[F_{k}: \mathbb{Q}\right]-1\right)$, and $q=p^{d}$ as before. The only difference with the unramified case concerns the quantity $\left(\Delta_{A}\right)^{1 /\left(\left[F_{k}: \mathbb{Q}\right] d\right)}$ which is not bounded along $F_{k} / \mathbb{Q}$. Put $n=\left[F_{k}: \mathbb{Q}\right]$. We have

$$
\frac{1}{d n} \log \Delta_{A}=d \log \operatorname{rd}_{F}+\frac{|S|}{n} f(e-1) \log p .
$$

Then, a good parameter $t>0$ does exist when

$$
\log p>\left(e-\frac{1}{2}\right) \log d+\frac{1}{2}(e-1) \log p+O(e),
$$

i.e when $e<3$. Although there is no room when $e \geq 3$ (as noted in [11, §7.5.3]), we may construct maximal orders good codes over finite fields by using quaternion algebras.

Remark 31. - The existence of unramified towers with splitting conditions and small root discriminant is central in the asymptotic analysis of number field codes, especially when we look for codes over $\mathbb{F}_{p^{2}}$ with $p$ as small as possible (see for example [5]). For towers of number fields of small root discriminant see for example [6].

Remark 32. - When $d$ is even, our last computation shows how to construct asymptotically good additive codes for the sum-rank distance from algebras of degree $d$ with alphabet $\mathrm{M}_{d / 2}\left(\mathbb{F}_{p^{2}}\right)$.

\section{References}

[1] A. Borel, Harish-Chandra, Arithmetic subgroups of algebraic groups. Ann. of Math. (2) $\mathbf{7 5}$ (1962) 485-535.

[2] N. Bourbaki, Groupes et Algèbres de Lie, Chapitres 7 et 8, Springer, 2017.

[3] D. Bump, Lie Groups, Graduate Texts in Math. 225, Springer, 2013.

[4] V.D. Goppa, Codes on algebraic curves, Soviet Math. Dokl. 24 (1981), 170-172.

[5] V. Guruswami, Construction of codes from number fields, IEEE Transactions on Information Theory 49 (3) (2003), 594-603.

[6] F. Hajir and C. Maire, Tamely ramified towers and discriminant bounds for number fields II, Journal of Symbolic Computation 33 (2002), 415-423.

[7] Z.-G. Hu and K.-H. Yan, The Weyl Integration Model for KAK decomposition od Reductive Lie Group, Arxiv, 2015.

[8] A.W. Knapp, Representation Theory of semisimple Groups, Princeton Landmarks in Mathematics, Princeton University Press, Princeton, NJ, 2001.

[9] H.W. Lenstra, Codes from algebraic number fields, In: M. Hazewinkel, J.K. Lenstra, L.G.C.T Meertens (eds), Mathematics and computer science II, Fundamental contributions in the Netherlands since 1945, CWI Monograph 4, pp. 95-104, North-Holland, Amsterdam, 1986.

[10] I.G. Macdonald, The volume of a compact Lie group, Inventiones Math. 56 (1980), 93-95.

[11] C. Maire and F. Oggier, Maximal order codes over number fields, Journal of Pure and Applied Algebra 227 (7) (2018), 1827-1858. 
[12] J. Martinet, Tours de corps de corps de classes et estimations de discriminants, Invent. Math. 44 (1978), 65-73.

[13] U. Martínez-Peñas, Skew and linearized Reed-Solomon codes and maximum sum rank distance codes over any division ring, Journal of Algebra, 504 (2018), 587-612.

[14] A. Page, Méthodes explicites pour les groupes arithmétiques, PhD Thesis, Bordeaux (France), 2014.

[15] The PARI Group, PARI/GP version 2.6.1, http://pari.math.u-bordeaux.fr/.

[16] V. Platonov and A. Rapinchuk, Algebraic Groups and Number Theory, Pure and Applied Math. Series 139, Academic Press Inc, Harcourt Brace and Compagny Publishers, 1994.

[17] G. Prasad, Volumes of S-arithmetic quotients of semi-simple groups, Publ. Math. IHES 69 (1989), 91-114.

[18] I. Reiner, Maximal Orders, London Math. Society Monographes New Series 28, Oxford Science Publications, 2003.

[19] M. Tsfasman, S. Vlăduţ and D. Nogin, Algebraic Geometric Codes: Basic Notions, Mathematical Surveys and Monographs 139, AMS, 2007.

September 1, 2020

Christian Maire, Institut FEMTO-ST, Univ. Bourgogne Franche-Comté, 15B Av. des Montboucons, F-25030 Besançon cédex, France

- E-mail : christian.maire@univ-fcomte.fr

Aurel Page, INRIA, Univ. Bordeaux, CNRS, IMB, UMR 5251, F-33400 Talence, France

E-mail : aurel.page@inria.fr 